\title{
Obstrução intestinal causada por Diverticulite de Meckel: Relato de caso
}

\author{
Meckel's Diverticulitis as cause of Intestinal Obstruction: Case report
}

Richard Raphael Borges Tavares Vieira ${ }^{\dagger *}$, Ana Beatriz Esteves Batista ${ }^{\dagger \S}$, Jair Jhonatam de Sá Silva ${ }^{\dagger *}$, Breno Barros Alves $^{\dagger}$, Joaquim Ferreira de Paula ${ }^{\star}$, Carlos Augusto Marques Batista ${ }^{\star 8}$

Como citar esse artigo. Vieira, R.R.B.T; Batista, A.B.E.; Silva, J.J.S.; Alves, B.B.; de Paula, J.F.; Batista, C.A.M. Obstrução intestinal causada por Diverticulite de Meckel: Relato de caso. Revista de Saúde. 2019 Jan./Jun.; 10 (1): 38-43.

\section{Resumo}

O Divertículo de Meckel é a anomalia congênita mais comum do trato gastrointestinal, incidindo em aproximadamente $2 \%$ da população em geral. Embora a maioria dos pacientes permaneça assintomática, ele pode se apresentar com uma variedade grande de manifestações clínicas quando surge algum tipo de complicação, geralmente na forma de inflamação, obstrução ou sangramento, tornando amplo o rol de diagnósticos diferenciais. Seu diagnóstico é difícil e ainda permanece como um grande desafio porque a maioria dos exames complementares mostra somente alterações decorrentes das complicações, sem precisão em estabelecer que estas ocorram nesta anomalia, e geralmente ocorre durante a cirurgia. $\mathrm{O}$ tratamento nos achados acidentais / incidentais, assim como nos casos manifestados com complicação, é ainda controverso e motivo de dúvidas. No presente estudo descreve-se um caso de diverticulite de Meckel como causa de obstrução do íleo pelo processo inflamatório, onde a técnica de diverticulectomia foi empregada com sucesso no tratamento, garantindo uma boa evolução pós-operatória.

Palavras-chave: Abdome agudo; Obstrução intestinal; Divertículo de Meckel; Diverticulectomia

\section{Introdução}

O Divertículo de Meckel é a anomalia congênita mais comum do trato gastrointestinal $1^{1-12,14,15}$. A sua incidência na população em geral é de aproximadamente $2 \%$ e embora a maioria dos pacientes permaneça assintomática ${ }^{1-3,11,13,14}$, o risco de complicação ocasionando sintomas nestes indivíduos é de 4-6 $\%^{1-3,12,13}$. É um divertículo verdadeiro e situado entre 30 a $180 \mathrm{~cm}$ da válvula ileocecal, na borda antimesentérica do íleo ${ }^{1-9,11-15}$. Pode se apresentar com uma variedade grande de manifestações clínicas quando surge algum tipo de complicação ${ }^{1-4,6-9,13,14}$.

Vários métodos de exames complementares de diagnóstico são citados na literatura, porém, a maioria deles mostra somente alterações decorrentes das complicações ${ }^{1,3-8,11,12}$. Apesar dos avanços em imagem e tecnologia, o diagnóstico clínico do divertículo de Meckel sintomático / complicado é muitas vezes difícil de estabelecer com precisão e geralmente ocorre durante a cirurgia, daí a importância da laparotomia ou laparoscopia exploradora diagnóstica e terapêutica ${ }^{1,4-}$ 6,8-10,12,15. O tratamento do divertículo de Meckel nos achados acidentais / incidentais é controverso, mas no divertículo sintomático indica-se a diverticulectomia ou ressecção intestinal $1^{1-7,9-15}$.

O objetivo deste trabalho é relatar um caso raro de divertículo de Meckel complicado com diverticulite aguda e obstrução intestinal, diagnosticado e tratado

Afiliação dos autores: $\uparrow$ Centro de Ensino Superior de Valença - Fundação Dom André Arcoverde (CESVA-FAA), Valença, RJ;

\ Hospital Escola Luiz Gioseffi Jannuzzi - Faculdade de Medicina de Valença (HELJG-FMV), Valença, RJ;

§ Liga Acadêmica de Cirurgia - Faculdade de Medicina de Valença (LAC-FMV), Valença, RJ

* Email de correpondência: rrbtv@hotmail.com 
no Serviço de Cirurgia Geral do Hospital Escola Luiz Gioseffi Jannuzzi da Faculdade de Medicina de Valença (HELGJ-FMV) e fazer uma revisão de literatura sobre o assunto. Foi submetido projeto ao Comitê de Ética de Pesquisa do Centro de Ensino Superior de Valença através da plataforma Brasil, sendo aprovado com número de parecer 2.826.213. Após isso, feita busca de artigos científicos em periódicos indexados ao PubMed, LILACS e Scielo e seleção dos mais relevantes para confrontar com os achados de nosso caso.

\section{Relato de Caso}

Homem, 37 anos, dá entrada no HELGJFMV relatando início de dor abdominal difusa tipo cólica, que não melhorou com antiespasmódicos, há aproximadamente uma semana. Evoluiu com manutenção da dor abdominal, alteração do hábito intestinal com parada de eliminação de gases e fezes, vômitos e distensão abdominal. Refere que já apresentou episódios semelhantes no passado, havia feito uma cirurgia prévia, etilista e tabagista.

Ao exame, apresentava sinais vitais normais (PA $=110 \times 80 \mathrm{mmHg}, \mathrm{FC}=72 \mathrm{bpm}, \mathrm{FR}=16 \mathrm{irpm}$ ) e fácies de dor; abdome distendido, doloroso à palpação e sem sinais de irritação peritonial, sem massa palpável ou visceromegalias, sinal de Blumberg negativo e peristalse diminuída.

Hemograma completo, função renal, EAS e amilase estavam todos normais. A rotina radiológica para abdome agudo (RRAA) mostrava alças de intestino delgado edemaciadas, distendidas, com níveis hidroaéreos e pouco gases em cólon e reto (Figura 1). A tomografia abdominal (TC) mostrou afilamento abrupto em íleo proximal, promovendo distensão intestinal a montante e níveis hidroaéreos, configurando processo obstrutivo de suboclusão intestinal, aparentemente correspondendo à hérnia interna (Figura 2).

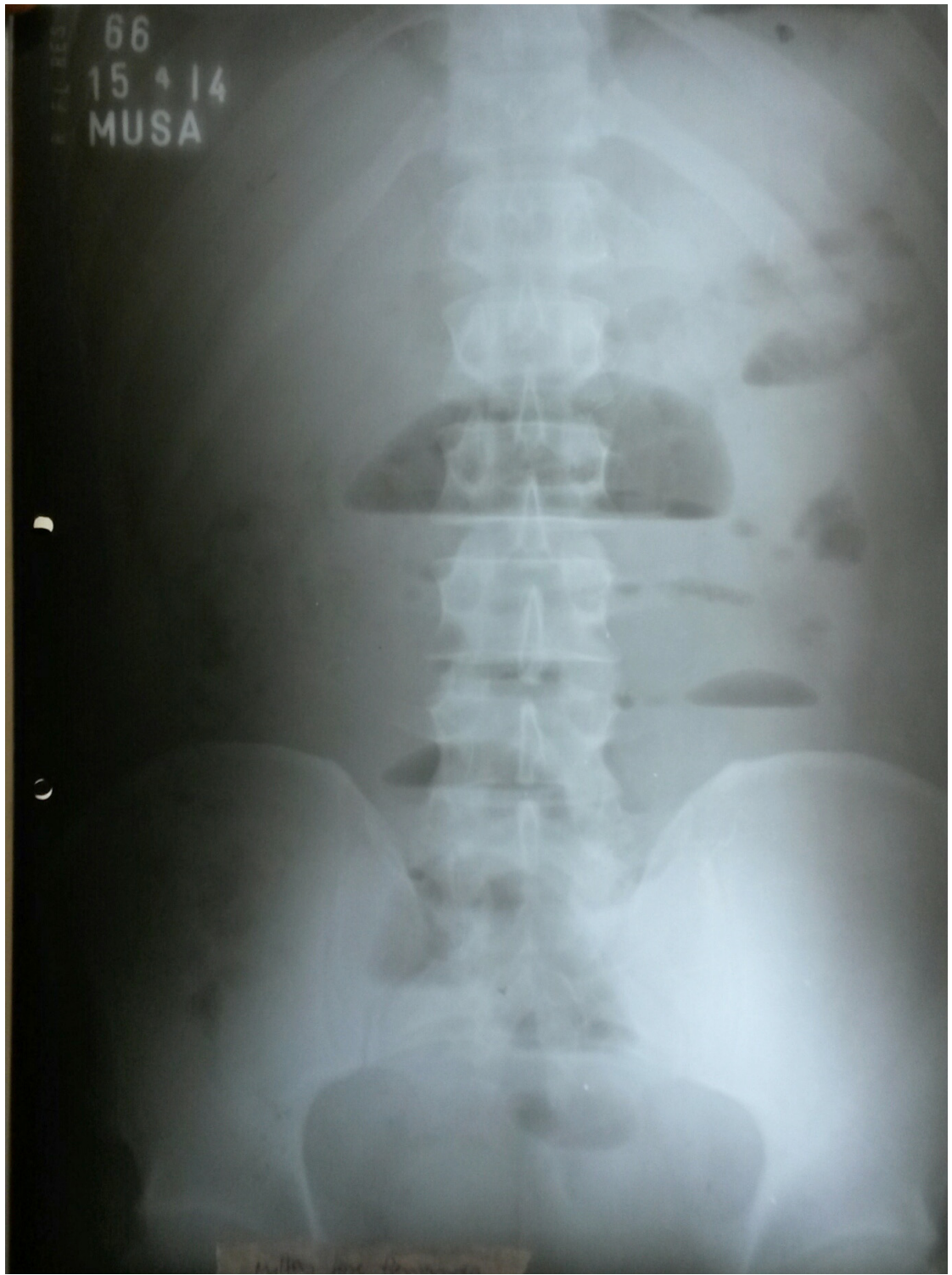

Figura 1. Radiografia de abdome em ortostase mostrando níveis hidroaéreos (autoria própria). 


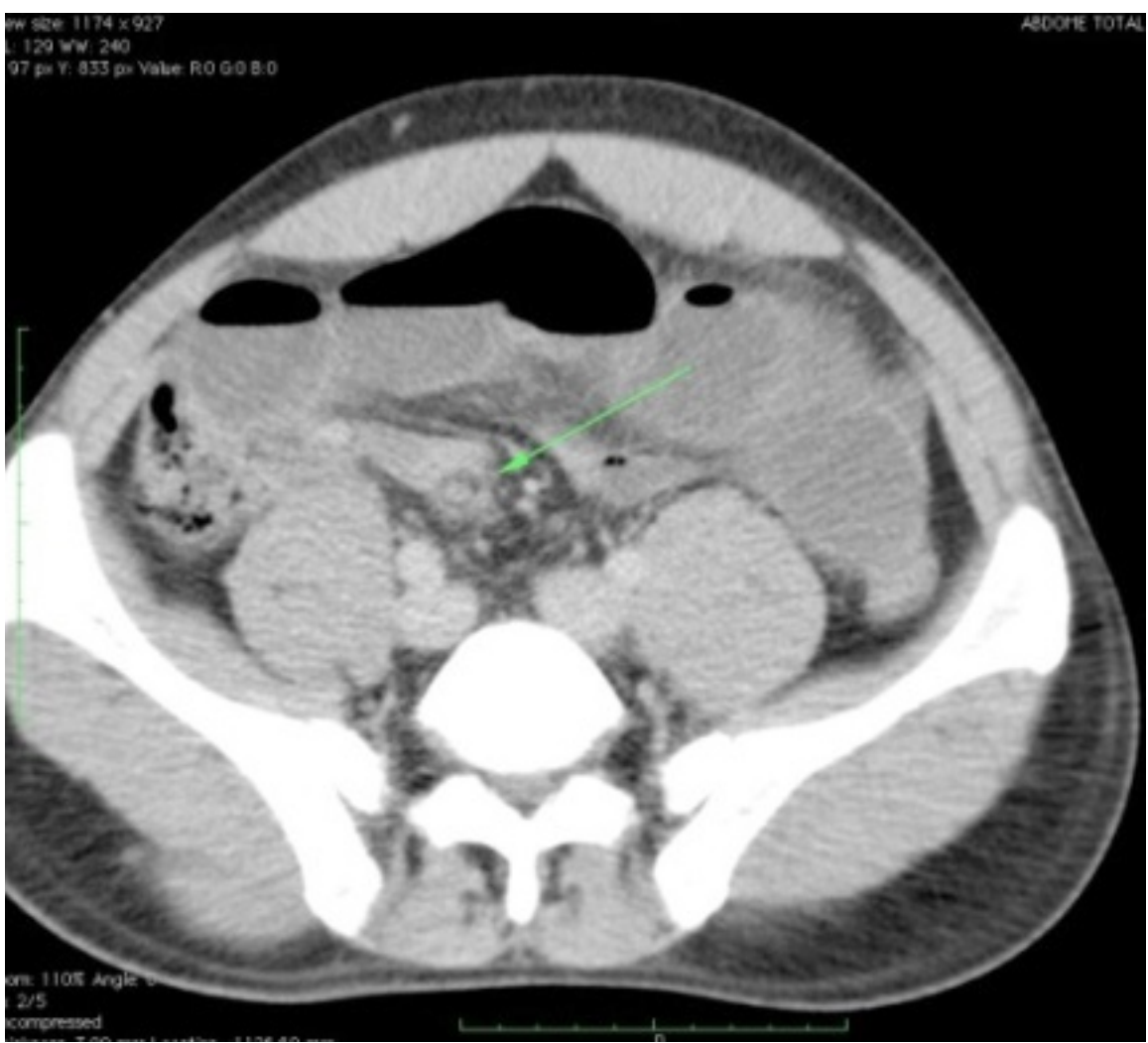

Figura 2. TC de abdome evidenciando também níveis hidroaéreos, além de distenção de intestino delgado e afilamento de íleo (autoria própria).

Foi internado em observação clínica no Serviço de Cirurgia Geral. O tratamento inicial consistiu de jejum, hidratação venosa, analgesia e cateterismo nasogástrico, que apresentou débito de $1.150 \mathrm{~mL} / 24$ horas. Após 48 horas, sem evidências de melhora do quadro abdominal que continuava distendido e doloroso, e o débito da sonda nasogástrica alto, optou-se por laparotomia exploradora mediana, por diagnóstico pré-operatório de obstrução intestinal por hérnia interna.

No inventário cavitário foi encontrado líquido serohemorrágico e divertículo de Meckel dobrado sobre o mesentério do íleo, distando aproximadamente $90 \mathrm{~cm}$ da válvula ileocecal, medindo $4,0 \times 1,5 \mathrm{~cm}$, com sinais inflamatórios em ponta e aderido à alça ileal proximal à sua adjacência, onde provocava acotovelamento e estreitamento; os segmentos intestinais à montante apresentavam edema importante, distensão e infiltrado hemorrágico. Foi realizado diverticulectomia e fechamento da cavidade (Figuras 3 e 4).

Evoluiu nos primeiros dias de pós-operatório com distensão abdominal e débito alto de sonda nasogástrica, sendo iniciada dieta líquida no $7^{\circ}$ dia com boa aceitação. Recebeu alta hospitalar no $12^{\circ}$ dia. Teve acompanhamento ambulatorial sem intercorrências, recebendo alta definitiva com 40 dias de pós-operatório. $\mathrm{O}$ anatomopatológico revelou Divertículo de Meckel com mucosa do tipo entérica exibindo diverticulite aguda com áreas de reação gigantocelular tipo corpo estranho.

\section{Discussão}

O Divertículo de Meckel foi descrito em 1598 por Fabricius Hildanus e em 1809 Johann Friedrich Meckel foi o primeiro a descrever sua anatomia e embriologia ${ }^{4,6-}$ 8,11,12,14. Littré em 1742 descreveu um divertículo de Meckel estrangulado em uma hérnia inguinal ${ }^{7,8}$.

É a anomalia congênita mais comum do trato gastrointestinal, ocorrendo em $1 \%-4 \%$ da população ${ }^{1-}$ $12,14,15$. Sua incidência pode estar aumentada em recémnascidos com outras anomalias, tais como: fenda palatina, pâncreas anular, atresia de esôfago e anorretal, entre outras ${ }^{7}$. Atinge igualmente ambos os sexos, porém, quando sintomático, o homem é mais acometido ${ }^{3-}$ $5,7,8,12,13$.

Representa um vestígio remanescente, por fechamento incompleto ou falha na absorção, do conduto onfalomesentérico ou vitelino entre a $7^{\mathrm{a}}$ e $8^{\mathrm{a}}$ semana de gestação-12,14,15. É em 90\% - 98\% a anormalidade mais encontrada, podendo estar associado a outros achados como fístula enterocutânea, seio umbilical, cisto do ducto onfalomesentérico e persistência de um cordão fibroso $^{4,8,11,12,15}$.

É um divertículo intestinal verdadeiro e normalmente está situado entre 30 a $180 \mathrm{~cm}$ da válvula ileocecal, na borda antimesentérica do íleo ${ }^{1-9,11-15}$. Geralmente é um divertículo curto e de base larga ${ }^{4,7,8,10}$. 


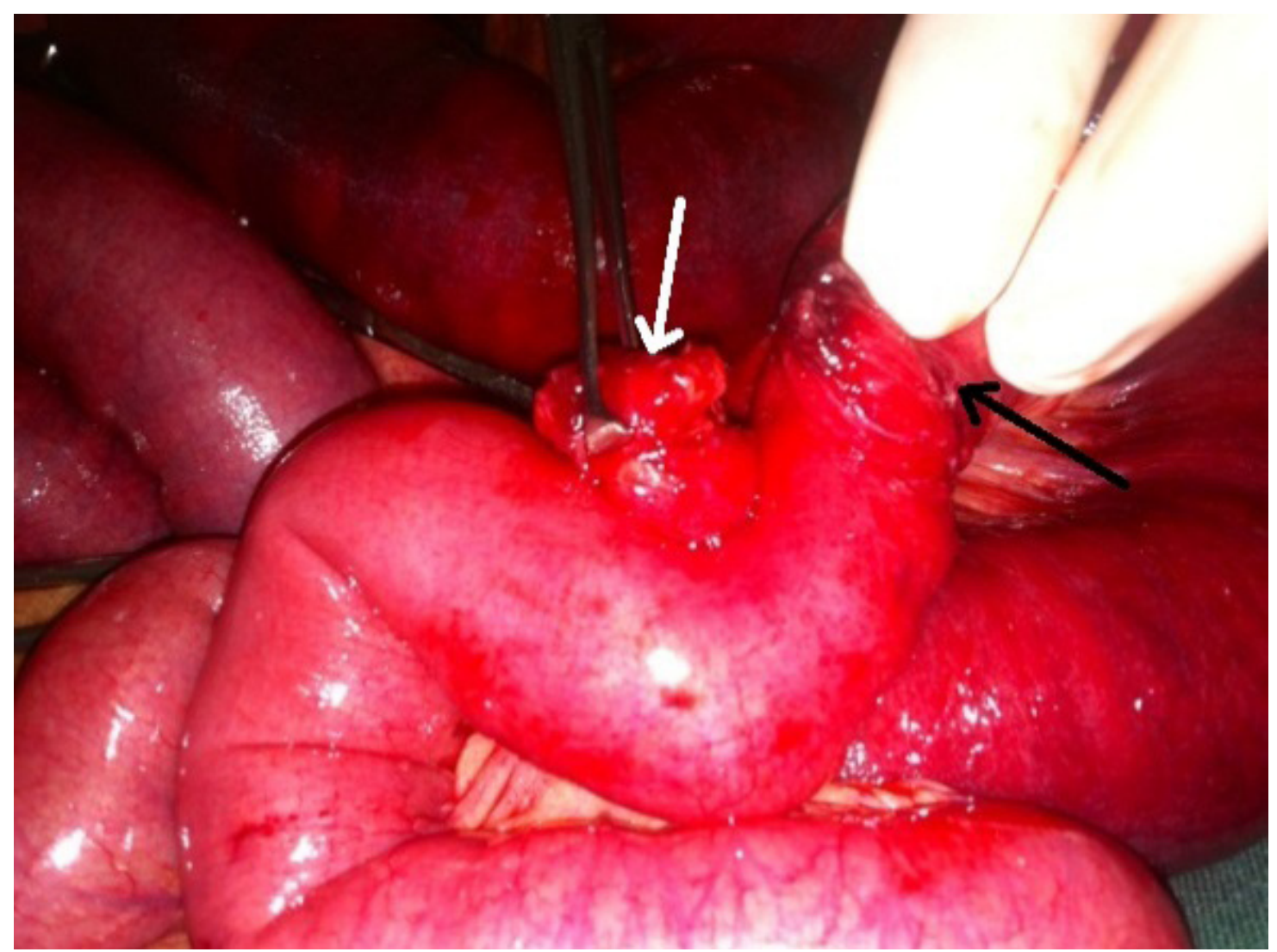

Figura 3. Divertículo de Meckel inflamado em seta branca promovendo estreitamento de alça ileal em seta preta (autoria própria).

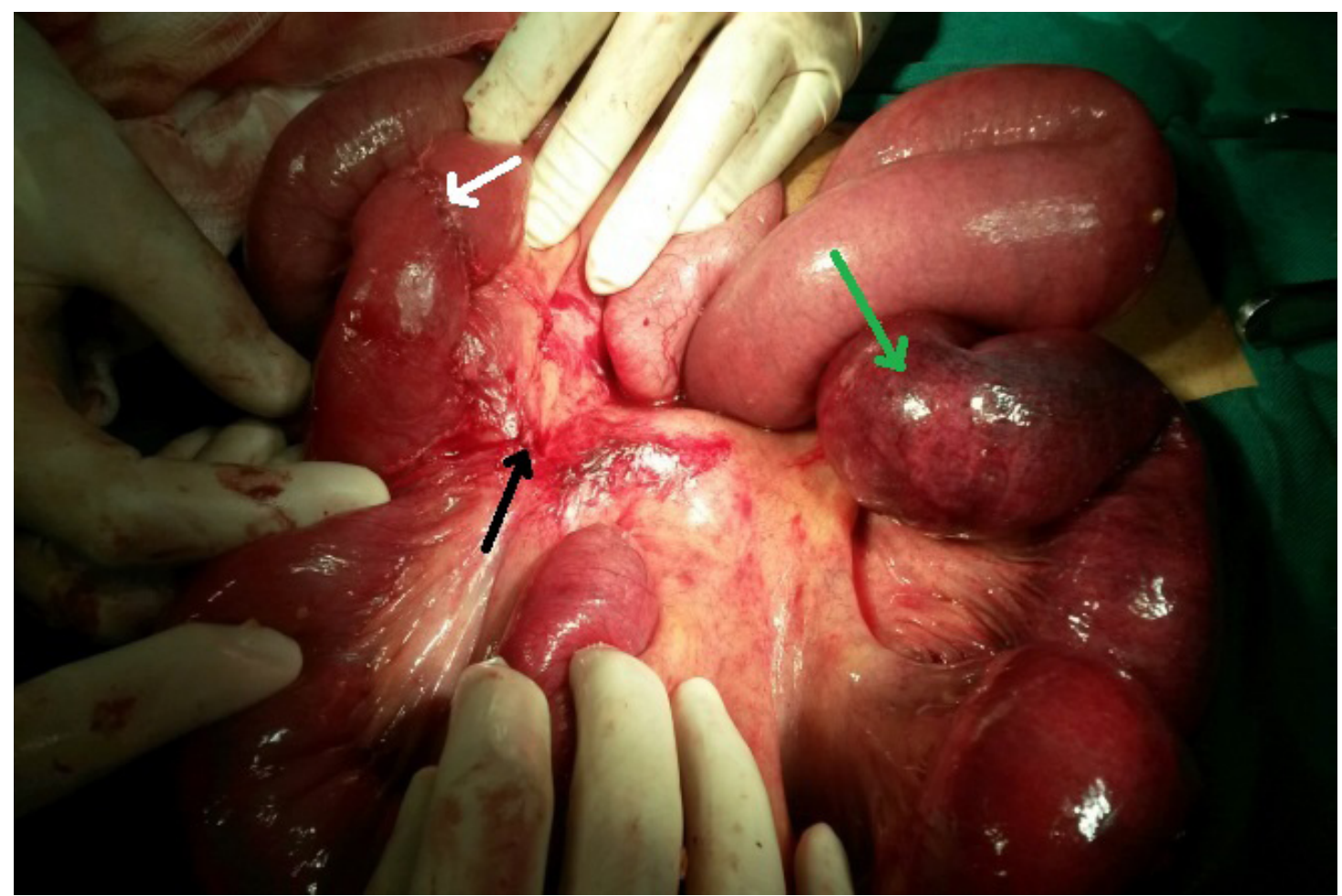

Figura 4. Região suturada em seta branca após diverticolectomia, local de aderência do divertículo em mesentério ileal em seta preta e infiltrado hemorrágico em seta verde (autoria própria). 
Pode ainda conter tecidos ectópicos em 30\% $50 \%$ dos casos, como mucosa gástrica $(60 \%-85 \%)$, pancreática $(5 \%-16 \%)$ e menos comumente, mucosa do cólon, endometriose e tecido hepatobiliar, os quais são responsáveis por complicações como hemorragia, úlcera péptica crônica e perfuração $0^{3-9,11-13,15}$. A transformação maligna da mucosa diverticular é rara e a histologia pode mostrar tumor carcinoide (44\%), GIST ou adenocarcinoma (16\%), entre outros ${ }^{7,12}$.

$\mathrm{O}$ divertículo de Meckel tem evolução clínica assintomática e é um achado incidental durante laparotomias e laparoscopias $(0,14 \%-4,5 \%)$ ou necropsias $(0,3 \%-4 \%)^{1-4,7-9,12,13}$. A literatura estima em $4 \%$ - $6 \%$ a possibilidade de a doença complicar e se tornar sintomática ao longo da vida ${ }^{3-7,11-13,15}$.

Pode se apresentar com uma variedade grande de manifestações clínicas quando surge algum tipo de complicação ${ }^{1-4,6-9,14,15}$. Elas devem ser consideradas no diagnóstico diferencial de pacientes com causa indeterminada de abdome agudo obstrutivo ou inflamatório ${ }^{1,2,6,11,12,15}$.

São descritas várias complicações, tais como: hemorragia, intussuscepção, inflamação, perfuração (geralmente por corpo estranho), obstrução, volvo, úlcera péptica, neoplasia e enterólitos ${ }^{3,4,7-9,11-15}$. O sangramento é a complicação mais frequente em crianças abaixo de dois anos ${ }^{4,6-12,13,15}$, sendo a obstrução intestinal a segunda causa mais comum ${ }^{12}$. Nos adultos, em aproximadamente $36 \%$, é a obstrução intestinal ${ }^{2-}$ 3,4,6-12 e entre os vários mecanismos encontramos a estenose secundária à diverticulite crônica, torção do divertículo, hérnia interna, hérnia de Littré, enterolitos, volvo, intussuscepção íleo-ileal, bridas inflamatórias e fitobezoares ${ }^{1-5,7,9-12}$. A perfuração diverticular e a diverticulite aguda, que pode ocorrer em $13 \%$ - 31\%, mais comumente entre quarta e quinta décadas de vida, são de difícil diagnóstico e têm na apendicite aguda seu principal diagnóstico diferencial ${ }^{7,12,14}$.

O diagnóstico do divertículo de Meckel é difícil e ainda permanece como um grande desafio na prática médica ${ }^{7}$. Charles Mayo imortalizou uma frase quanto à dificuldade de diagnóstico do divertículo de Meckel: “o divertículo de Meckel é frequentemente suspeito, muitas vezes procurado, porém raramente encontrado" 7,13 .

Vários métodos de exames complementares são citados na literatura, tais como: RRAA, exame contrastado do intestino delgado, TC, USG, arteriografia, cintilografia com Tecnécio 99 pertecnetato (exame padrão-ouro quando mucosa gástrica heterotópica) e cápsula endoscópica ${ }^{1,3-8,11,12}$. Porém, a maioria dos exames complementares mostra somente alterações decorrentes das complicações; a RRAA mostra normalmente pneumoperitônio, distensão de alças intestinais e níveis hidroaéreos e a TC pode identificar melhor o local da obstrução ${ }^{5,7,9,10,13,14}$.

Apesar dos avanços em imagem e tecnologia, o diagnóstico clínico da forma sintomática/complicada é muitas vezes difícil de estabelecer com precisão $0^{1,3,4,8,11,12}$ e geralmente ocorre durante a cirurgia, daí a importância da laparotomia ou laparoscopia exploradora diagnóstica e terapêutica ${ }^{1,4-6,8-10,12,14}$. No nosso caso, a RRAA revelou achados inespecíficos comum às doenças agudas abdominais de natureza obstrutiva e inflamatória. A TC demonstrou sinais que sugeriam suboclusão intestinal no íleo proximal, sem, no entanto, ter responsabilizado o divertículo de Meckel como o causador do problema.

O tratamento nos achados acidentais/incidentais é ainda motivo de dúvidas e controverso, e suas indicações não estão bem estabelecidas ${ }^{1-4,7,11,13-15}$. Os autores que recomendam sua retirada alegam que é impossível mostrar que não haja mucosa heterotópica no seu interior e que ele não venha a ter complicações no futuro, indicando a cirurgia, principalmente, em jovens ${ }^{4,11,15}$ e também quanto ao possível desenvolvimento de neoplasia diverticular? ${ }^{7}$. Outros mostram que é baixa a taxa de sintomas e complicações, principalmente em idosos, justificando a não ressecção $0^{2,3,6,7,11}$. Shelat ${ }^{11} \mathrm{e}$ Goulart ${ }^{15}$ citaram autores que defendem a não retirada do divertículo diagnosticado incidentalmente devido haver pouca chance do mesmo se tornar sintomático e causar complicações e que essa taxa de complicações é menor que a morbimortalidade cirúrgica. Naufel $\mathrm{Jr}^{14}$ relatou uma série de artigos que mostram que os benéficos do procedimento cirúrgico superam a morbimortalidade da intervenção. Kundra ${ }^{13}$ relatou que a taxa de complicações da ressecção em pacientes assintomáticos é de aproximadamente $1 \%$ - 6\%, comparando com uma taxa de $5 \%-7 \%$ de potenciais complicações ao longo da vida. Outros autores ainda defendem uma abordagem seletiva, indicando a ressecção nas seguintes situações: homens com menos de 50 anos, divertículo maior que dois centímetros, detecção de características anormais no interior do divertículo ${ }^{11}$.

Kundra ${ }^{13}$ conclui em seu artigo que como não há um protocolo aceito para o tratamento da forma assintomática, o procedimento deve ser avaliado caso a caso, levando em consideração a história clínica, o tamanho e a aparência do divertículo e os achados da palpação que possam sugerir mucosa espessada ou anormal, considerando também os riscos e benefícios do procedimento.

O tratamento definitivo do divertículo de Meckel sintomático é a cirurgia, através de laparotomia ou laparoscopia, sendo a laparotomia ainda a via de acesso mais utilizada ${ }^{1-7,9-12,15}$. Quando possível, a abordagem laparoscópica, segundo alguns autores, é a melhor opção e a de eleição, visto que mesmo apresentando limitações, é segura e eficiente, apresenta menos complicações e menor tempo de recuperação que a laparotomia; embora seu uso dependa das condições do paciente, experiência do cirurgião e disponibilidade dos instrumentais ${ }^{6,7,10,12}$. A literatura também relata o 
procedimento cirúrgico realizado simultaneamente por laparoscopia e via transumbilical aberta, o que permitiria a palpação e melhor avaliação do divertículo de Meckel e suas complicações ${ }^{12}$.

A extensão da ressecção, diverticulectomia ou enterectomia, é ainda controversa, e é guiada pelo tipo de complicação e do achado intra-operatório, tais como: sangramento, presença de tecido ectópico, necrose, perfuração, abscesso peri-diverticular ou suspeita de neoplasia ${ }^{1-7,10-12,14,15}$. Na diverticulectomia, a realização de sutura mecânica da base diverticular é uma opção preferível, porque evita a abertura do tubo digestivo ${ }^{12,14,15}$. Martinez ${ }^{3}$ ainda cita a possibilidade de ordenha em direção ao ceco ou enterotomia para retirada de enterolitos, que causam obstrução intestinal. No caso em questão, como as alterações inflamatórias eram restritas à ponta do divertículo, decidiu-se apenas pela sua ressecção, como relatado por outros autores ${ }^{1,2,6,8,11}$.

É relatado taxa de $7 \%-11 \%$ de complicações pós-operatórias no tratamento cirúrgico do divertículo de Meckel $^{1,6,9,12,13}$

\section{Considerações finais}

O diagnóstico do divertículo de Meckel é ainda um desafio. O tratamento definitivo é cirúrgico, sendo ainda controverso quando de achados acidentais / incidentais, porém mediante suas complicações, tanto a diverticulectomia quanto a enterectomia podem ser realizadas, quer por via laparotômica ou laparoscópica.

\section{Referências Bibliográficas}

1. Maia DS, Ferreira-Júnior M, Viegas RG, Silva EEC, Oliveira PTV, Silva RCA, Caetano AJ, Ferreira NG. Obstrução intestinal por divertículo de Meckel. ABCD Arq Bras Cir Dig. 2013; 26(3): 244-245

2. Lima IS, Leal AT, Houly RLS, Alves AS. Divertículo de Meckel como causa de obstrução intestinal: Relato de um caso. Rev Bras Colo-Proct. 1989; $9(1): 25-27$

3. Martinez CAR, Priolli DG, Palma RT, Waisberg J, Margarido NF. Obstrução intestinal por enterolito formado em divertículo de Meckel: Relato de caso. Rev Bras Coloproct. 2004; 24(2):165-169.

4. Massoni Neto LM, Hinkel BB, Dói A, Alcântara PSM. Obstrução intestinal devido à enterólitos em divertículo de Meckel: Relato de caso. Rev Med (São Paulo). 2007; 86(3):155-62.

5. Dick OW, Müller V, Breunig JA, Braga DC. Hérnia interna causada por divertículo de Meckel: Relato de caso. Revista AMRIGS (Porto Alegre) 2003; 47(4):280-282

6. Brásio R, Malaquias R, Gil I, Rama N, Ferraz S, Faria V. Divertículo de Meckel: Um caso de hemorragia digestiva baixa. Rev Port Coloproct. 2015; $12(1): 45-48$.

7. Araújo LM, Araujo FM, Alves ACS, Monteiro ACF, Paula BC, Xavier DSS, Alves EVS, Sezko IA. Divertículo de Meckel: Revisão de literatura. Rev Med Minas Gerais. 2014; 24(1):93-97.

8. Porto GG, Rebello FV, Vasconcelos CN. Divertículo de Meckel: Um relato de caso. Braz J Surg Clin Res. 2016; 14(3):57-59.

9. Curvello CFA. Obstrução intestinal por divertículo de Meckel: Relato de caso. Rev Cien FMC 2016; 11(1):15-17.

10. Marquardt Filho N, Rasche L, Horbach VC, Seibert AP, Reffati D, Navarini D. Divertículo de Meckel obstruido por fitobezoar. Rev Col Bras Cir 2017; 4:1-3.

11. Shelat VG, Li KK, Rao A, Guan TS. Meckel's diverticulitis causing small bowel obstruction by a novel mechanism. Clin Prac. 2011; 1:102-104.

12. Sharma RK, Jain VK. Emergency surgery for Meckel's diverticulum. World J Emerg Surg. 2008; 3:27-35.

13. Kundra DN, Agarwal R, Gupta A, Vashishth S. Incidental Asymptomatic Meckel's Diverticulum: A Therapeutic Dilemma. Int J Med Sci. 2015; 2(5): $1-2$

14. Naufel Júnior CR, Varaschim M, Naufel AMO, Perraro EIC. Divertículo de Meckel perfurado por palito de dente. Rev Col Bras Cir. 2016; 4:1-3.

15. Goulart A, Pereira R, Leão P, Gomes C, Carvalho A. Divertículo de Meckel perfurado por palito: relato de caso clínico. Rev Port Cir. 2011; 2(17): 41-4. 